\title{
Efficient unidirectional nanoslit couplers for surface plasmons
}

\author{
F. LÓPEZ-TEJEIRA ${ }^{1}$, SERGIO G. RODRIG0 ${ }^{1}$, L. MARTÍN-MORENO ${ }^{1 *}$, F. J. GARCÍA-VIDAL ${ }^{2}$, E. DEVAUX³, \\ T. W. EBBESEN ${ }^{3}$, J. R. KRENN ${ }^{4}$, I. P. RADKO ${ }^{5}$, S. I. BOZHEVOLNYI5 ${ }^{5}$, M. U. GONZÁLEZ ${ }^{6 \dagger}$, J. C. WEEBER ${ }^{6}$ \\ AND A. DEREUX6
}

\author{
'Departamento de Física de la Materia Condensada-ICMA, Universidad de Zaragoza, E-50009 Zaragoza, Spain \\ ${ }^{2}$ Departamento de Física Teórica de la Materia Condensada, Universidad Autónoma de Madrid, E-28049 Madrid, Spain \\ ${ }^{3}$ Laboratoire de Nanostructures, ISIS, Université Louis Pasteur, F-67000 Strasbourg, France \\ ${ }^{4}$ Institute of Physics, Karl Franzens University, Universitätsplatz 5, A-8010 Graz, Austria \\ ${ }^{5}$ Department of Physics and Nanotechnology, Aalborg University, DK-9220 Aalborg, Denmark \\ ${ }^{6}$ Laboratoire de Physique de l'Université de Bourgogne, UMR CNRS 5027, F-21078 Dijon, France \\ ${ }^{\dagger}$ Present address: ICFO-Institut de Ciències Fotòniques, Mediterranean Technology Park, E-08860 Castelldefels, Barcelona, Spain \\ *e-mail: Imm@unizar.es
}

The emerging field of plasmonics is based on exploiting the coupling between light and collective electronic excitations within conducting materials known as surface plasmons. Because the so-called surface plasmon polariton (SPP) modes that arise from this coupling are not constrained by the optical diffraction limit, it is hoped that they could enable the construction of ultracompact optical components ${ }^{1,2}$. But in order that such potential can be realized, it is vital that the relatively poor light-SPP coupling be improved. This is made worse by the fact that the incident light that is conventionally used to launch SPPs in a metal film $^{3-6}$ is a significant source of noise, unless directed away from a region of interest, which then decreases the signal and increases the system's size. Back-side illumination of subwavelength apertures in optically thick metal films $s^{7-13}$ eliminates this problem but does not ensure a unique propagation direction for the SPP. We propose a novel back-side slit-illumination method that incorporates a periodic array of grooves carved into the front side of a thick metal film. Bragg reflection enhances the propagation of SPPs away from the array, enabling them to be unidirectionally launched from, and focused to, a localized point.

A picture of the proposed surface plasmon polariton (SPP) launcher is shown in Fig. 1. A periodic array of one-dimensional indentations is fabricated at the (output) metal surface close and parallel to the illuminated slit. The design of this device is based on two facts. The first one is that the reflection of SPPs by a periodic array of indentations presents maxima at the low- $\lambda$ edges of the plasmonic bandgaps ${ }^{14-16}$. For subwavelength indentations, the spectral locations of these edges can be obtained by folding the dispersion relation of SPPs for a flat metal surface into the first Brillouin zone, satisfying the following expression:

$$
k_{\mathrm{p}} P=m \pi,
$$

where $P$ is the period of the array, $k_{\mathrm{p}}$ holds for the in-plane plasmon wavevector and $m$ is the band index. Remarkably, although the

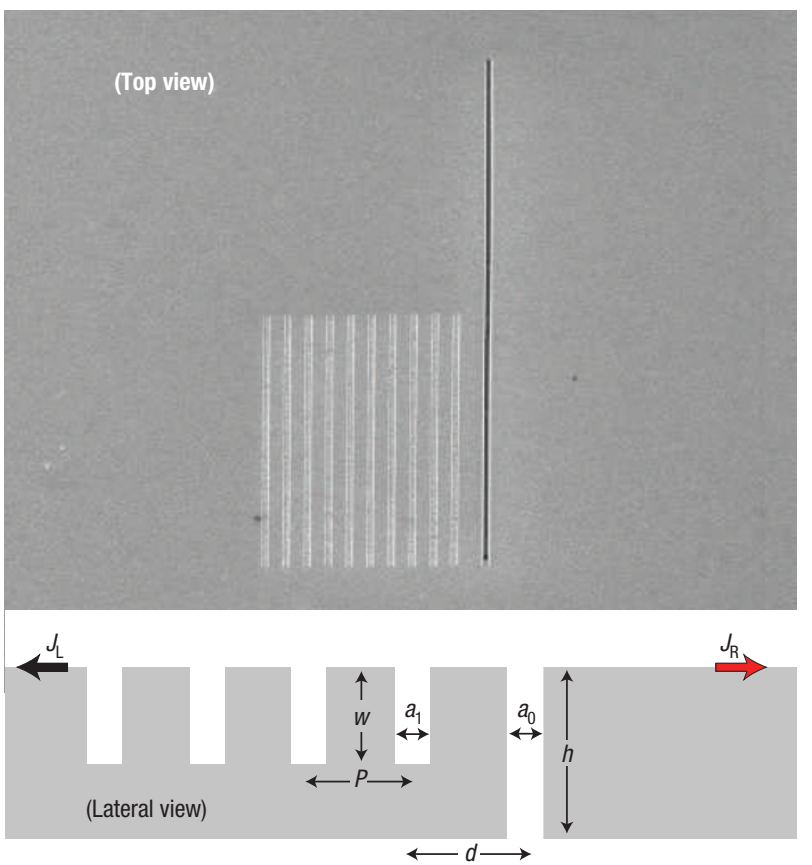

Figure 1 Scanning electron micrographs and schematic diagrams of the structures investigated. The parameters used in the definition of the slit, grooves and metal film are also shown. $J_{R}$ and $J_{L}$ are the current energy densities for rightand left-propagating SPPs.

reflectance maxima depend on the groove geometry (width and depth) and the number of grooves, their spectral locations do not.

The second fact is that the phase picked up by the SPP on reflection is just $m \pi$, precisely at the condition given by 


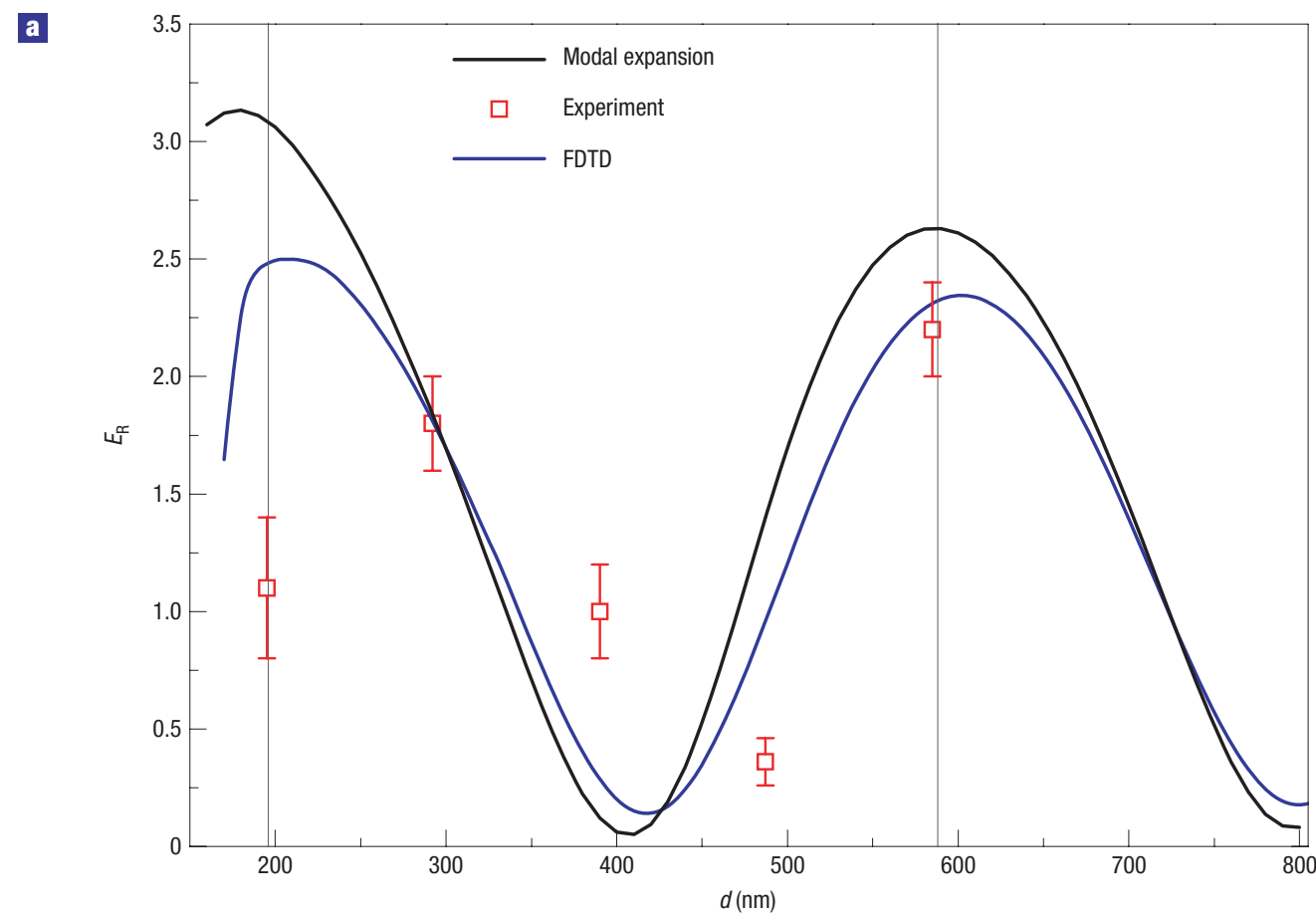

b
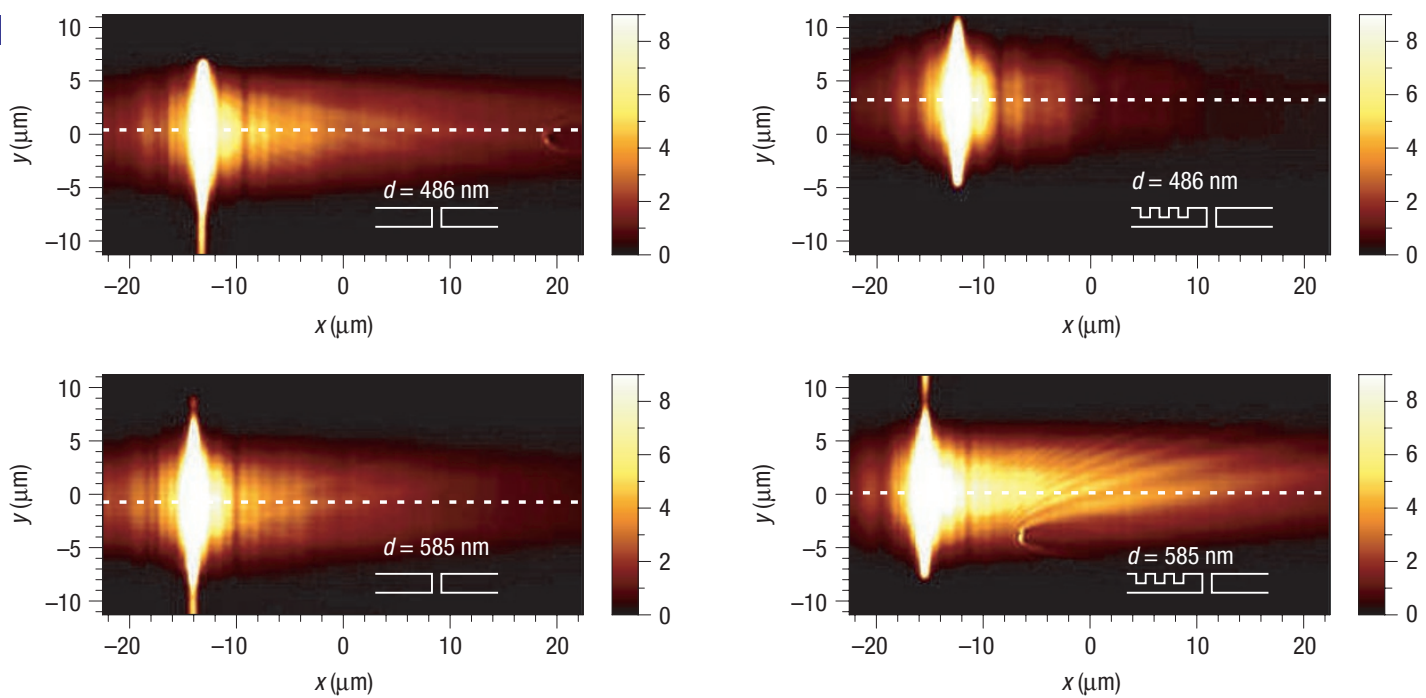

Figure 2 Theoretical and experimental results for the SPP launcher at wavelength $\lambda=\mathbf{8 0 0} \mathbf{n m}$. a, Dependence with slit-array distance of the efficiency ratio $E_{\mathrm{R}}$. The geometrical parameters defining the system are: slit length $30 \mu \mathrm{m}$, slit and groove widths $a=160 \mathrm{~nm}$, groove depth $w=100 \mathrm{~nm}$ and array period $P=390 \mathrm{~nm}$. The vertical lines mark the positions of $E_{\mathrm{R}}$ maxima according to equation (2). The error bars for the experimental results represent the maximum variation in $E_{\mathrm{R}}$ with respect to its mean value, from measurements on different structures with the same nominal parameters. b. Photon scanning tunnelling micrographs recorded at $\lambda=800 \mathrm{~nm}$ for two different slit-grating distances. Left column: isolated slit. Right column: slit + grating. $(x, y)$ define the plane of the top metal surface, with $y$ being the slit axis.

equation (1), as obtained by the modal expansion developed in ref. 15. Using these two results, a very simple scheme for the efficient unidirectional launching of SPPs can be predicted. For a given frequency, by choosing $P$ such that the condition given by equation (1) is fulfilled, an SPP emerging from the slit to the left side will be mainly backscattered. The interference of this reflected SPP with the one leaving the slit to the right can be tuned by adjusting the separation, $d$, between the slit and the first groove of the array (defined centre to centre). The total phase difference, $\phi$, between the two interfering SPPs will be the phase picked on reflection plus the one associated with their different path lengths along the metal:

$$
\phi=2 k_{\mathrm{p}} d+m \pi .
$$

According to equation (2), destructive or constructive interference should occur for those $\phi$ values equal to odd or even multiples of $\pi$, respectively. In these latter cases, the device would behave as an efficient source for unidirectional SPPs. 
Note that equation (2) is based on two main simplifications. First, the previous discussion is based on the reflection of SPPs by a groove array, whereas the electromagnetic (EM) fields radiated by the slit are, at short distances, more complex ${ }^{15}$. Second, equation (2) does not take into account the radiation from the grooves back into the slit, whereas, in principle, EM fields at all openings should be self-consistently calculated ${ }^{17}$.

To check the validity of equation (2) we have carried out numerical calculations by means of both modal expansion ${ }^{15}$ and finite-difference time-domain (FDTD) ${ }^{18}$ methods. FDTD is virtually exact for this type of one-dimensional structures, as very small grid sizes can be used. On the other hand, the modal expansion treats approximately the finite conductivity of the metal but provides a very compact representation for the EM fields, favouring the physical interpretation and, in some simple cases, the calculation of analytical expressions. We characterize the efficiency of the slit + groove system as an SPP launcher by the 'efficiency ratio', $E_{\mathrm{R}}$, defined as the quotient between the current intensity of the right-propagating SPP $\left(J_{\mathrm{R}}\right)$ with and without the grooves. Strictly speaking, $E_{\mathrm{R}}$ provides the efficiency of the output side of the device; the total efficiency, defined as the percentage of laser beam energy transferred onto the plasmon channel, depends also on lateral beam size, substrate dielectric constant, metal film width, corrugation on the input side and so on. Note also that $E_{\mathrm{R}}>2$ implies that, in the corrugated structure, the right-propagating SPP carries more current than the total SPP current (left- plus rightmoving) in the single-slit case, so some of the power radiated out of plane is redirected onto the SPP channel.

The model system is a nanoslit SPP launcher perforated on a gold film ${ }^{19}$, designed to operate at a wavelength of $800 \mathrm{~nm}$, inside the near-infrared range of the EM spectrum. We consider an array of ten grooves with a period $P=390 \mathrm{~nm}$, obtained from equation (1) with $m=1$. The depth of the grooves is chosen to be $w=100 \mathrm{~nm}$, whereas the width of both the grooves and the slit is $a=160 \mathrm{~nm}$, which are typical experimental parameters. Figure $2 \mathrm{a}$ shows the calculated dependence of $E_{\mathrm{R}}$ with distance $d$. In this figure, the vertical lines mark the locations of maximum interference predicted by equation (2). The agreement between the modal expansion and FDTD results is excellent, except for the behaviour at very short distances $(d \approx 2 a)$, owing to the cross coupling between the slit and the first groove through the vertical walls, which is neglected within the modal expansion. More importantly, the locations of maximum $E_{\mathrm{R}}$ are accurately predicted by equation (2), which allows us to design SPP launchers without elaborate numerical calculations.

Note that $E_{\mathrm{R}}$ would be 4 if the whole amplitude of the left-going SPP could be added constructively to the right-going one, whereas in our simulations a smaller value is always obtained. Calculations with the modal expansion show that this is due to the out-of-plane scattering of the left-going SPP by the array of grooves. The effect on $E_{\mathrm{R}}$ of both damping across the flat gold surface and partial transmission across the finite array plays a very minor role for the considered parameters.

To test experimentally our proposal, several samples were prepared with a focused ion beam in 300-nm-thick gold films for different values for $d$, with all other geometrical parameters being the same as in the previous calculations. Each sample consists of a single long slit flanked by a finite periodic groove array that extends over only half of the slit length (see Fig. 1a). This sample design allows the quantitative experimental study of the SPP launching efficiency, as the 'isolated' slit (upper part) can be used as an in-chip reference. The set of samples was imaged by a photon scanning tunnelling microscope making use of an incident focused beam illumination for frequencies in the $[765,800] \mathrm{nm}$ interval. Owing to specific features of the experimental set-up used for measurements
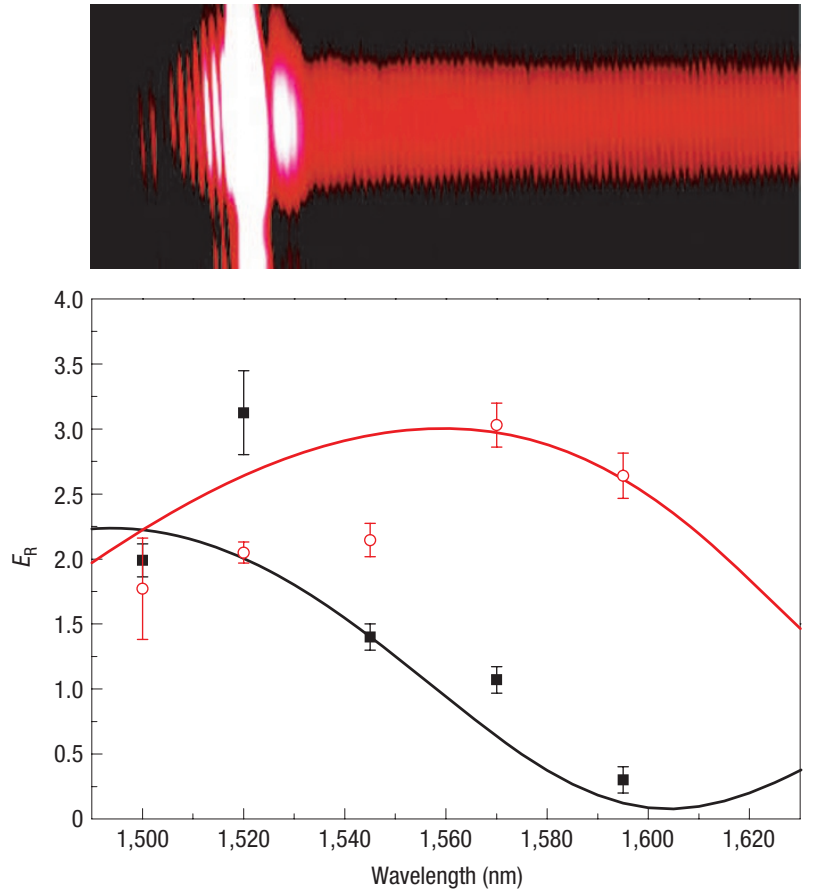

Figure 3 Experimental results and modal expansion calculations for the spectral dependence of $E_{\mathrm{R}}$ at the telecom range. Two samples were considered. In both, the slit length is $50 \mu \mathrm{m}$ and the slit and groove widths are 400 and $200 \mathrm{~nm}$, respectively, with groove periodicity $P=750 \mathrm{~nm}$, film thickness $h=300 \mathrm{~nm}$ and groove depth $w=100 \mathrm{~nm}$. In one sample, the slit-grating distance

$d=3 P / 2=1,125 \mathrm{~nm}$ (experiment: black squares; theory: black curve), whereas in the other $d=3 P / 4=562 \mathrm{~nm}$ (experiment: red circles; theory: red curve). The image in the upper panel shows grating-induced increased coupling for $d=3 P / 2$ at $\lambda=1,520 \mathrm{~nm}$. (Size $=70 \times 26 \mu \mathrm{m}^{2}$.) The error bars reflect the maximum variation in $E_{\mathrm{R}}$ obtained from different measurements for the same configuration with independent adjustments of the illumination. Weak periodic modulation of the strong SPP beam propagating to the right (upper panel) is due to its interference with an SPP wave reflected by a remote auxiliary structure.

in the optical regime, the incident laser beam was directed on the sample (attached to a prism) under an angle of $43^{\circ}$ with respect to the normal. However, it should be noted that the choice of angle of incidence is not critical for the spatial distribution of transmitted energy, as a subwavelength slit in an optically thick metal film transmits only in the fundamental mode. For each distance, $d$, a pair of images was recorded by scanning at a constant distance of about $60-80 \mathrm{~nm}$ from the sample surface. The first image of the pair, corresponding to the SPP launching by a single slit, is obtained by focusing the laser beam on the upper part of the slit. For the second image, the laser beam is moved to the lower part to collect the data for the slit + grating case. Image pairs for $d=585 \mathrm{~nm}$ and for $d=486 \mathrm{~nm}$ are shown in Fig. 2b. Figure $2 \mathrm{~b}$ clearly shows that the grating increases the intensity of the right-propagating SPP for $d=585 \mathrm{~nm}$, whereas for $d=486 \mathrm{~nm}$ this intensity is greatly reduced. To quantify this effect, an average longitudinal crosscut of each image is obtained by using 20 longitudinal cross-cuts, corresponding to different coordinates along the slit axis. Then, the relative position of the two average cross-cuts composing each image pair is adjusted so that the saturated areas (that is, the signal taken right on top of the slit) are superimposed. Finally, the experimental efficiency ratio, $E_{\mathrm{R}}$, is extracted by averaging the ratio between the two curves along the longitudinal cross-cut. 

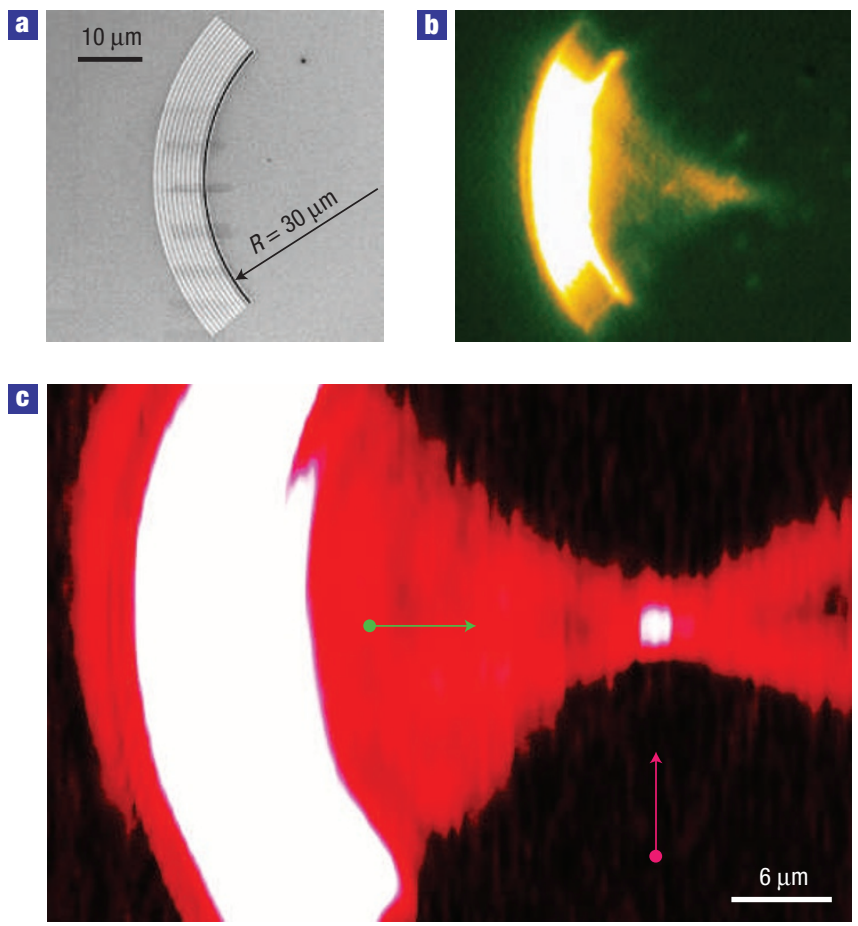

d

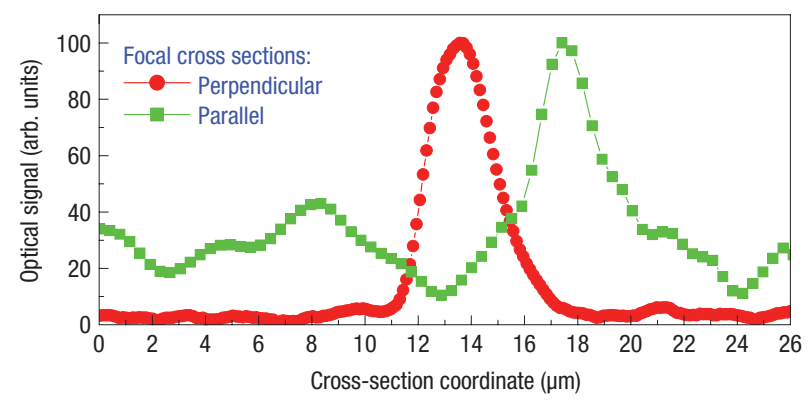

Figure 4 Simultaneous unidirectional SPP excitation and focusing using a curved slit flanked with concentric periodic grooves. a, Scanning electron micrograph of the structure characterized by slit and groove widths of 400 and $200 \mathrm{~nm}$, respectively, groove periodicity $750 \mathrm{~nm}$, groove depth $100 \mathrm{~nm}$ and slit-groove distance $1,125 \mathrm{~nm}$. Film thickness is $280 \mathrm{~nm}$, curvature radius is $30 \mu \mathrm{m}$ and slit chord length is $40 \mu \mathrm{m}$. b,c, Far-field image recorded with a charge-coupled device camera (b) and the corresponding near-field optical image

$\left(\right.$ size $\left.=48 \times 32 \mu \mathrm{m}^{2}\right)(\mathbf{c})$, both being obtained when the curved slit was illuminated at normal incidence with radiation at the telecom wavelength of $1,520 \mathrm{~nm}$.

d, Normalized cross-sections obtained from the optical image (c) by dissecting the SPP focal spot perpendicular and parallel to the SPP propagation direction, as indicated on the image (c) by the red and green arrows, respectively. The dots on the arrows mark the origin of the coordinate system for each cross-section.

Figure 2a shows experimental results for $E_{\mathrm{R}}$ for the five different samples fabricated. The agreement between the experimental data and the theoretical predictions is quite remarkable (especially when taking into account that each experimental point corresponds to a different sample), showing that the presence of the grating modulates the coupling into the right-propagating SPP.

We have also designed similar samples for efficient unidirectional SPP excitation at telecom wavelengths, up-scaling the grating period and its separation from the slit. In this case, normal incidence back-side illumination is allowed by the experimental set-up and used in all experiments.
Figure 3 (upper panel) shows a typical near-field optical image, featuring a strong SPP beam propagating away from the slit in the direction opposite to the grating and thereby demonstrating unidirectional SPP excitation. In this wavelength range, the long SPP propagation length $(\approx 200 \mu \mathrm{m})$ allows a simpler determination of $E_{\mathrm{R}}$ as the power ratio between the SPP beams, estimated far away $(\approx 50 \mu \mathrm{m})$ from the slit, obtained from the near-field optical images taken by focusing the laser beam at two different vertical positions.

The efficiency ratio determined in this way exhibited a significant dispersion due to inaccuracy in the illuminating laser beam adjustment. Consequently, several series of measurements were carried out, conducting independent adjustments for each sample and wavelength. Averaged results and estimated errors for the spectral dependence of $E_{\mathrm{R}}$ are shown in Fig. 3. As can be seen, the comparison between theory and experiments is satisfactory: for the case of the sample with $d=P+P / 2=1,125 \mathrm{~nm}, E_{\mathrm{R}}$ decreases as the wavelength increases (with the only exception of a sharp peak at $1,520 \mathrm{~nm})$, evolving from a favourable regime $\left(E_{\mathrm{R}} \approx 2\right)$ to one in which SPP coupling is clearly diminished by the grating $\left(E_{\mathrm{R}}<1\right)$. On the other hand, $E_{\mathrm{R}} \approx 2$ all over the range for the sample with $d=P-P / 4=562 \mathrm{~nm}$, as predicted by the modal expansion calculation.

Another application of the system proposed in this paper is the focusing of SPPs, creating local field enhancement (a 'hot spot') at a given location. Focusing of SPPs has been achieved through the interaction of SPPs with curved surface corrugations $s^{20-24}$. The proposed approach for the localized unidirectional excitation of an SPP beam can be generalized to focus SPPs with an increased efficiency while blocking the propagation away from the focus. Importantly, in these curved structures, the rigorous modelling of SPP excitation needed for optimization of the focusing would be difficult, whereas the relation given by equation (2) still provides simple design rules. As a proof of principle, in this paper we present focusing at telecom wavelengths by milling a curved slit along with the corresponding grating grooves (Fig. 4a), using a similar configuration to that illustrated with the near-field optical image shown in Fig. 3. The effect of SPP beam focusing was clearly seen already at the stage of far-field adjustment (using a microscope arrangement with an infrared charge-coupled device camera) owing to weak out-of-plane SPP scattering by surface roughness (Fig. 4b). The typical near-field optical image obtained at $\lambda=1,520 \mathrm{~nm}$ demonstrates efficient focusing of a launched SPP beam at the centre of slit curvature, with a spot size of $3 \times 3 \mu \mathrm{m}^{2}$ (estimated from the cross-cuts shown in Fig. 4d). More generally, the design of other more complicated curved structures based on these principles can be predicted, allowing, for example, the excitation of SPP beams propagating in different directions and focused at different locations.

Received 1 September 2006; accepted 29 January 2007; published 8 April 2007.

References

1. Barnes, W. L., Dereux, A. \& Ebbesen, T. W. Surface plasmon subwavelength optics. Nature 424 824-830 (2003).

2. Ozbay, E. Plasmonics: Merging photonics and electronics at nanoscale dimensions. Science 311, 189-193 (2006)

3. Otto, A. Exitation of nonradiative surface plasma waves in silver by the method of frustrated total reflection. Z. Phys. 216, 398-410 (1968).

4. Lamprecht, B. et al. Surface plasmon propagation in microscale metal stripes. Appl. Phys. Lett. 79, 51-53 (2001).

5. Ritchie, R. H., Arakawa, E. T., Cowan, J. J. \& Hamm, R. N. Surface-plasmon resonance effect in grating diffraction. Phys. Rev. Lett. 21, 1530-1533 (1968).

6. Ditlbacher, H. et al. Fluorescence imaging of surface plasmon fields. Appl. Phys. Lett. 80, 404-406 (2002).

7. Sönnichsen, C. et al. Launching surface plasmons into nanoholes in metal films. Appl. Phys. Lett. 76, $140-142(2000)$.

8. Devaux, E., Ebbesen, T. W., Weeber, J. C. \& Dereux, A. Launching and decoupling surface plasmons via micro-gratings. Appl. Phys. Lett. 83, 4936-4938 (2003).

9. Yin, L. et al. Surface plasmons at single nanoholes in Au films. Appl. Phys. Lett. 85, 467-469 (2004). 10. Popov, E. et al. Surface plasmon excitation on a single subwavelength hole in a metallic sheet. Appl. Opt. 44, 2332-2337 (2005) 
11. Agrawal, A., Cao, H. \& Nahata, A. Excitation and scattering of surface plasmon-polaritons on structured metal films and their application to pulse shaping and enhanced transmission. New J. Phys. 7, 249 (2005).

12. Chang, S. H., Gray, S. K. \& Schatz, G. C. Surface plasmon generation and light transmission by isolated nanoholes and arrays of nanoholes in thin metal films. Opt. Express 13, 3150-3165 (2005).

13. Lalanne, P., Hugonin, J. P. \& Rodier, C. Theory of surface plasmon generation at nanoslit apertures. Phys. Rev. Lett. 95, 263902 (2005).

14. Bozhevolnyi, S. I., Boltasseva, A., Sondergaard, T., Nikolajsen, T. \& Leosson, K. Photonic bandgap structures for long-range surface plasmon polaritons. Opt. Commun. 250, 328-333 (2005).

15. López-Tejeira, F., García-Vidal, F. J. \& Martín-Moreno, L. Scattering of surface plasmons by one-dimensional periodic nanoindented surfaces. Phys. Rev. B 72, 161405(R) (2005).

16. González, M. U. et al. Design, near-field characterization, and modeling of $45^{\circ}$ surface-plasmon Bragg mirrors. Phys. Rev. B 73, 155416 (2006).

17. Martín-Moreno, L., García-Vidal, F. J., Lezec, H. J., Degiron, A. \& Ebbesen, T. W. Theory of highly directional emission from a single subwavelength aperture surrounded by surface corrugations. Phys. Rev. Lett. 90, 167401 (2003).

18. Taflove, A. \& Hagness, S. C. Computational Electrodynamics: The Finite-Difference Time-Domain Method (Artech House, Boston, 2000)

19. Vial, A., Grimault, A., Macias, D., Barchesi, D. \& de la Chapelle, M. Improved analytical fit of gold dispersion: Application to the modeling of extinction spectra with a finite-difference time-domain method. Phys. Rev. B 71, 085416 (2005).
20. Nomura, W., Ohtsu, M. \& Yatsui, T. Nanodot coupler wih a surface plasmon polariton condenser for optical far/near-field conversion. Appl. Phys. Lett. 86, 181108 (2005).

21. Yin, L. et al. Subwavelength focusing and guiding of surface plasmons. Nano Lett. $\mathbf{5}$ 1399-1402 (2005).

22. Liu, Z et al. Focusing surface plasmons with a plasmonic lens. Nano Lett. 5, 1726-1729 (2005).

23. Offerhous, H. L. et al. Creating focused plasmons by noncollinear phasematching on functional gratings. Nano Lett. 5, 2144-2148 (2005).

24. Steele, J. M., Liu, Z., Wang, Y. \& Zhang, X. Resonant and non-resonant generation and focusing of surface plasmons with circular gratings. Opt. Express 14, 5664-5670 (2006).

\section{Acknowledgements}

Financial support by the EC under Project FP6-2002-IST-1-507879 (Plasmo-Nano-Devices) is gratefully acknowledged. We thank J. Dintinger and J.-Y. Laluet for technical assistance. Correspondence and requests for materials should be addressed to L.M.-M.

\section{Competing financial interests}

The authors declare no competing financial interests.

Reprints and permission information is available online at http://npg.nature.com/reprintsandpermissions/ 